\title{
Hopf Bifurcation of an Improved SLBS Model under the Influence of Latent Period
}

\author{
Chunming Zhang, ${ }^{1}$ Wanping Liu, ${ }^{2}$ Jing Xiao, ${ }^{1}$ and Yun Zhao ${ }^{1}$ \\ ${ }^{1}$ School of Information Engineering, Guangdong Medical College, Dongguan 523808, China \\ ${ }^{2}$ College of Computer Science, Chongqing University, Chongqing 400044, China
}

Correspondence should be addressed to Chunming Zhang; chunfei2002@163.com

Received 12 June 2013; Accepted 15 August 2013

Academic Editor: Fazal M. Mahomed

Copyright ( 92013 Chunming Zhang et al. This is an open access article distributed under the Creative Commons Attribution License, which permits unrestricted use, distribution, and reproduction in any medium, provided the original work is properly cited.

\begin{abstract}
A model applicable to describe the propagation of computer virus is developed and studied, along with the latent time incorporated. We regard time delay as a bifurcating parameter to study the dynamical behaviors including local asymptotical stability and local Hopf bifurcation. By analyzing the associated characteristic equation, Hopf bifurcation occurs when the time delay passes through a sequence of critical values. A formula for determining the direction of the Hopf bifurcation and the stability of bifurcating periodic solutions is given by using the normal form method and center manifold theorem. Finally, illustrative examples are given to support the theoretical results.
\end{abstract}

\section{Introduction}

With the rapid development of computer technologies and network applications, the Internet has become a powerful mechanism for propagating computer virus. Because of this, computers connected to the Internet become much vulnerable to digital threats.

It is quite urgent to understand how computer viruses spread over the Internet and to propose effective measures to cope with this issue. To achieve this goal, and in view of the fact that the spread of virus among computers resembles that of biological virus among a population, it is suitable to establish dynamical models describing the propagation of computer viruses across the Internet by appropriately modifying epidemic models [1].

Based on the fact that infectivity is one of the common features shared by computer viruses and their biological counterparts [2], some classic epidemic models were established for computer virus propagation, such as the SIRS model [3-7], the SEIR model [8, 9], the SEIRS model [10], the SEIQV model [11], and the SEIQRS model [12]. In [13-15] the authors made the following assumptions.

(H1) All computers connected to the Internet are partitioned into three compartments: susceptible computers (S-computers), infected computers that are latent ( $L$-computers), and infected computers that are breaking out ( $B$-computers).

(H2) All newly connected computers are virus-free.

(H3) External computers are connected to the Internet at constant rate $\delta$. Meanwhile, internal computers are disconnected from the Internet at rate $\delta$.

(H4) Each virus-free computer is infected by a virulent computer at constant rate $\beta$, and the ratio of previously virus-free computers that are infected exactly at time $t$ is $\beta S(L+B)[16]$.

(H5) Breaking-out computers are cured at constant rate $\gamma$.

(H6) Latent computers break out at constant rate $\alpha$.

According to the above assumptions, the authors of [14, 15] proposed the proposed the following SLBS model, which is formulated as

$$
\begin{gathered}
\dot{S}=\delta-\beta S(L+B)+\gamma B-\delta S, \\
\dot{L}=\beta S(L+B)-\alpha L-\delta L, \\
\dot{B}=\alpha L-\gamma B-\delta B .
\end{gathered}
$$

It is well known that some computer viruses would delay a period to break out after the computer is infected. However, 
the above model fails to consider the concrete time of the delay. Thus, this paper aims to establish a model to incorporate the unconsidered factor, by adding a delay item to the above model. First, we give another assumption as (H7): $L$-computers turn out to be $B$-computers with constant time delay $\tau$.

According to the above assumptions (H1)-(H7), the new model with time delay is formulated as

$$
\begin{gathered}
\dot{S}=\delta-\beta S(L+B)+\gamma B-\delta S, \\
\dot{L}=\beta S(L+B)-\alpha L(t-\tau)-\delta L, \\
\dot{B}=\alpha L(t-\tau)-\gamma B-\delta B .
\end{gathered}
$$

Here, we let $S(t), L(t)$, and $B(t)$ represent the percentage of $S$-, $L$-, and $B$-computers in all internal computers at time $t$, respectively. Then we get $S(t)+L(t)+B(t) \equiv 1$ and consider the following equivalent two-dimensional subsystem:

$$
\begin{gathered}
\dot{L}=\beta(1-L-B)(L+B)-\alpha L(t-\tau)-\delta L, \\
\dot{B}=\alpha L(t-\tau)-\gamma B-\delta B .
\end{gathered}
$$

The initial conditions of (3) are given by $L(\theta)=\phi_{1}(\theta)>0$, $B(\theta)=\phi_{2}(\theta)>0$, and $\theta \in[-\tau, 0]$, where $\left(\phi_{1}(\theta), \phi_{2}(\theta)\right) \epsilon$ $C\left([-\tau, 0], R_{+}^{2}\right)$, the Banach space of the continuous functions mapping the interval $[-\tau, 0]$ into $R_{+}^{2}=\left\{\left(x_{1}, x_{2}\right): x_{i} \geq 0, i=\right.$ $1,2\}$.

The remainder of this paper is organized as follows. In Section 2, the stability of trivial solutions and the existence of Hopf bifurcation are discussed. In Section 3, a formula for determining the direction of Hopf bifurcation and the stability of bifurcating periodic solutions will be given by using the normal form and center manifold theorem introduced by Hassard et al. in [17]. In Section 4, numerical simulations aimed at justifying the theoretical analysis will be reported.

\section{Stability of the Equilibria and Existence of Hopf Bifurcation}

This section is intended to study model (3) theoretically, by analyzing the stability of its solutions and the existence of Hopf bifurcation. For the convenience of the following description, we define the basic reproduction number of system (3) as

$$
R_{0}=\frac{\beta(\alpha+\gamma+\delta)}{(\alpha+\delta)(\gamma+\delta)} .
$$

We have the following result with respect to the stable state of system (3).

Theorem 1. Consider system (3) with $\tau=0$. Then the unique virus-free equilibrium $E_{0}=(0,0)$ is globally asymptotically stable if $R_{0}<1$, whereas $E_{0}$ becomes unstable and the unique positive equilibrium $E_{*}=\left((\gamma+\delta)\left(1-1 / R_{0}\right) /(\alpha+\delta+\gamma), \alpha(1-\right.$ $\left.1 / R_{0}\right) /(\alpha+\delta+\gamma)$ ) is locally asymptotically stable if $R_{0}>1$.

The proof is omitted here (see [14] for details).
The linearized equations of (3) are as follows:

$$
\begin{gathered}
\dot{L}=\left[-\beta+\frac{2 \beta}{R_{0}}-\delta\right] L+\left[-\beta+\frac{2 \beta}{R_{0}}\right] B-\alpha L(t-\tau), \\
\dot{B}=\alpha L(t-\tau)-\gamma B-\delta B .
\end{gathered}
$$

The determinant of the Jacobian matrix of the system (5) at $E_{*}$ is given by $\left|y E-A_{1}-B_{1} e^{-y \tau}\right|=0$, where

$$
\begin{gathered}
A_{1}=\left[\begin{array}{cc}
-\beta+\frac{2 \beta}{R_{0}}-\delta & -\beta+\frac{2 \beta}{R_{0}} \\
0 & -\gamma-\delta
\end{array}\right], \\
B_{1}=\left[\begin{array}{cc}
-\alpha & 0 \\
\alpha & 0
\end{array}\right] .
\end{gathered}
$$

Let $d=-\beta+(2(\alpha+\delta)(\gamma+\delta)) /(\alpha+\gamma+\delta)$, and we can obtain the following characteristic equation:

$$
y^{2}+m_{1} y+m_{0}+\left(n_{1} y+n_{0}\right) e^{-y \tau}=0
$$

where $m_{1}=\gamma+2 \delta-d, m_{0}=(\gamma+\delta)(\delta-d), n_{1}=\alpha, n_{0}=$ $\alpha(\gamma+\delta-d)$.

Theorem 2. Suppose that $R_{0}>1, \gamma+\delta \geq \alpha$, and $(\gamma+\delta)^{2}(\delta-d)^{2}-\alpha^{2}(\gamma+\delta-d)^{2}<0$ hold; then the positive equilibrium $E_{*}$ is asymptotically stable for $\tau \in\left[0, \tau_{0}\right)$ and system (3) undergoes a Hopf bifurcation at $E_{*}$ when $\tau=\tau_{0}$.

Proof. Suppose that $y=i \omega(\omega>0)$ is a root of (7); then separating the real and imaginary parts of (7), we have

$$
\begin{gathered}
m_{1} \omega=n_{0} \sin \omega \tau-n_{1} \omega \cos \omega \tau, \\
\omega^{2}-m_{0}=n_{0} \cos \omega \tau+n_{1} \omega \sin \omega \tau .
\end{gathered}
$$

Adding up the squares of (8) yields

$$
\omega^{4}+\left(m_{1}^{2}-2 m_{0}-n_{1}^{2}\right) \omega^{2}+m_{0}^{2}-n_{0}^{2}=0 .
$$

Since $\gamma+\delta \geq \alpha$, we derive the following equations:

$$
\begin{gathered}
m_{1}^{2}-2 m_{0}-n_{1}^{2} \\
=(\gamma+2 \delta-d)^{2}-2(\gamma+\delta)(\delta-d)-\alpha^{2} \\
=(\delta-d)^{2}+(\gamma+\delta+\alpha)(\gamma+\delta-\alpha)>0, \\
m_{0}^{2}-n_{0}^{2}=(\gamma+\delta)^{2}(\delta-d)^{2}-\alpha^{2}(\gamma+\delta-d)^{2}<0 .
\end{gathered}
$$

Therefore, (9) exists as a unique positive solution $\omega_{0}$, and the characteristic equation (7) has a pair of pure imaginary roots $\pm i \omega_{0}$. By (8), we have

$$
\begin{aligned}
\tau_{n}= & \frac{1}{\omega_{0}} \arccos \frac{n_{0}\left(\omega_{0}^{2}-m_{0}\right)-m_{1} n_{1} \omega_{0}^{2}}{n_{0}^{2}+n_{1}^{2} \omega_{0}^{2}} \\
& +\frac{2 n \pi}{\omega_{0}}, \quad n=0,1,2, \ldots
\end{aligned}
$$


By Theorem 1 , for $\tau=0$, the positive equilibrium $E_{*}$ is locally asymptotically stable, and hence by Butler's Lemma [18], $E_{*}$ remains stable for $\tau<\tau_{0}$. Now, we need to show

$$
\left.\frac{\mathrm{d}(\operatorname{Re} y)}{\mathrm{d} \tau}\right|_{\tau=\tau_{0}}>0 .
$$

This will signify that there exists at least one eigenvalue with positive real part for $\tau>\tau_{0}$. Moreover, the conditions for Hopf bifurcation [19] are then satisfied yielding the required periodic solution. Now, by differentiating (9) with respect to $\tau$, we get

$$
\begin{aligned}
(2 y+ & \left.m_{1}+n_{1} e^{-y \tau}-\tau\left(n_{1} y+n_{0}\right) e^{-y \tau}\right) \frac{\mathrm{d} y}{\mathrm{~d} \tau} \\
& =y\left(n_{1} y+n_{0}\right) e^{-y \tau} .
\end{aligned}
$$

This gives

$$
\begin{aligned}
{\left[\frac{\mathrm{d} y}{\mathrm{~d} \tau}\right]^{-1} } & =\frac{2 y+m_{1}+n_{1} e^{-y \tau}-\tau\left(n_{1} y+n_{0}\right) e^{-y \tau}}{y\left(n_{1} y+n_{0}\right) e^{-y \tau}} \\
& =\frac{2 y+m_{1}}{y\left(n_{1} y+n_{0}\right) e^{-y \tau}}+\frac{n_{1}}{y\left(n_{1} y+n_{0}\right)}-\frac{\tau}{y} \\
& =\frac{y^{2}-m_{0}}{-y^{2}\left(y^{2}+m_{1} y+m_{0}\right)}+\frac{-n_{0}}{y^{2}\left(n_{1} y+n_{0}\right)}-\frac{\tau}{y} .
\end{aligned}
$$

Thus,

$$
\begin{gathered}
\operatorname{sign}\left\{\frac{\mathrm{d}(\operatorname{Re} y)}{\mathrm{d} \tau}\right\}_{\tau=\tau_{k}} \\
=\operatorname{sign}\left\{\operatorname{Re}\left[\frac{\mathrm{d} y}{\mathrm{~d} \tau}\right]^{-1}\right\}_{y=i \omega_{0}} \\
=\operatorname{sign}\left\{\operatorname{Re}\left[\frac{y^{2}-m_{0}}{-y^{2}\left(y^{2}+m_{1} y+m_{0}\right)}\right]\right. \\
=\operatorname{sign}\left\{\frac{\left(\omega_{0}^{2}+m_{0}\right)\left(\omega_{0}^{2}-m_{0}\right)}{\omega_{0}^{2}\left[\left(m_{0}-\omega_{0}^{2}\right)^{2}+\left(m_{1} \omega_{0}\right)^{2}\right]}\right. \\
\left.+\frac{n_{0}^{2}}{\omega_{0}^{2}\left(n_{0}^{2}+n_{1}^{2} \omega_{0}^{2}\right)}\right\} .
\end{gathered}
$$

Since $\left(m_{0}-\omega_{0}^{2}\right)^{2}+\left(m_{1} \omega_{0}\right)^{2}=n_{0}^{2}+n_{1}^{2} \omega_{0}^{2}$, we have that

$$
\begin{aligned}
& \operatorname{sign}\left\{\frac{\mathrm{d}(\operatorname{Re} y)}{\mathrm{d} \tau}\right\}_{\tau=\tau_{k}} \\
& =\operatorname{sign}\left\{\frac{\left(\omega_{0}^{2}+m_{0}\right)\left(\omega_{0}^{2}-m_{0}\right)}{\omega_{0}^{2}\left[\left(m_{0}-\omega_{0}^{2}\right)^{2}+\left(m_{1} \omega_{0}\right)^{2}\right]}+\frac{n_{0}^{2}}{\omega_{0}^{2}\left(n_{0}^{2}+n_{1}^{2} \omega_{0}^{2}\right)}\right\} \\
& =\operatorname{sign}\left\{\frac{\omega_{0}^{4}+n_{0}^{2}-m_{0}^{2}}{\omega_{0}^{2}\left(n_{0}^{2}+n_{1}^{2} \omega_{0}^{2}\right)}\right\} .
\end{aligned}
$$

As $m_{0}^{2}-n_{0}^{2}<0$, thus

$$
\left.\frac{\mathrm{d}(\operatorname{Re} y)}{\mathrm{d} \tau}\right|_{\tau=\tau_{0}}>0 .
$$

Therefore, the transversality condition holds and thus Hopf bifurcation occurs at $\tau=\tau_{0}$. The proof is complete.

\section{Direction of the Hopf Bifurcation}

In this section, we derive explicit formulae for computing the direction of the Hopf bifurcation and the stability of bifurcation periodic solution at critical value $\tau_{0}$ by using the normal form theory and center manifold reduction.

Letting $t=s \tau, u_{1}=L-L_{*}, u_{2}=B-B_{*}, u_{i}(t)=u_{i}(\tau t)$, and $\tau=\tau_{0}+\mu$, system (3) is transformed to an FDE as

$$
\dot{u}(t)=L_{v}\left(u_{t}\right)+f\left(\mu, u_{t}\right),
$$

where $u(t)=\left(u_{1}(t), u_{2}(t)\right)^{T} \in R^{2}, u_{t}(\theta)=u(t+\theta), \theta \in[0,1]$, $L_{v}: C \rightarrow R, f: R \times C \rightarrow R$,

$$
\begin{aligned}
& L_{v}\left(u_{t}\right) \\
& =\left(\tau_{0}+\mu\right) \\
& \quad \times\left[\begin{array}{cc}
-\beta+\frac{2(\alpha+\delta)(\gamma+\delta)}{\alpha+\gamma+\delta}-\delta & -\beta+\frac{2(\alpha+\delta)(\gamma+\delta)}{\alpha+\gamma+\delta} \\
0 & -\gamma-\delta
\end{array}\right] \\
& \quad \times\left[\begin{array}{c}
\varphi_{1}(0) \\
\varphi_{2}(0)
\end{array}\right]+\left(\tau_{0}+\mu\right)\left[\begin{array}{cc}
-\alpha & 0 \\
\alpha & 0
\end{array}\right]\left[\begin{array}{c}
\varphi_{1}(-1) \\
\varphi_{2}(-1)
\end{array}\right] \\
& =\left(\tau_{0}+\mu\right) A_{1}\left[\begin{array}{c}
\varphi_{1}(0) \\
\varphi_{2}(0)
\end{array}\right]+\left(\tau_{0}+\mu\right) B_{1}\left[\begin{array}{l}
\varphi_{1}(-1) \\
\varphi_{2}(-1)
\end{array}\right], \\
& f\left(v, u_{t}\right)=\left(\tau_{0}+\mu\right)\left[\begin{array}{c}
-\beta \varphi_{1}^{2}(0)-2 \beta \varphi_{1}(0) \varphi_{2}(0)-\beta \varphi_{2}^{2}(0) \\
0
\end{array}\right] .
\end{aligned}
$$

Using the Riesz representation theorem, there exists a function $\eta(\theta, \mu)$ of bounded variation for $\theta \in[0,1]$, such that

$$
L_{v} \varphi=\int_{-1}^{0} \mathrm{~d} \eta(\theta, \mu) \varphi(\theta), \quad \varphi \in C
$$

In fact, we can choose

$$
\eta(\theta, \mu)=\left(\tau_{0}+\mu\right) A_{2} \delta(\theta)+\left(\tau_{0}+\mu\right) B_{2} \delta(\theta+1),
$$

where $\delta(\theta)$ is Dirac delta function. In the following, for $\varphi \in$ $[0,1]$, we define

$$
\begin{gathered}
A(\mu) \varphi= \begin{cases}\frac{\mathrm{d} \varphi(\theta)}{\mathrm{d} \theta}, & \theta \in[-1,0), \\
\int_{-1}^{0} \mathrm{~d} \eta(s, \mu) \varphi(s), & \theta=0,\end{cases} \\
R(\mu) \varphi= \begin{cases}0, & \theta \in[-1,0), \\
f(\mu, \varphi), & \theta=0 .\end{cases}
\end{gathered}
$$


Then system (18) can be rewritten as

$$
\dot{u}(t)=A(\mu) u_{t}+R(\mu) u_{t},
$$

where

$$
\frac{\mathrm{d} u_{t}(\theta)}{\mathrm{d} \theta}=\frac{\mathrm{d} u(t+\theta)}{\mathrm{d} \theta}=\frac{\mathrm{d} u(t+\theta)}{\mathrm{d} t}=\frac{\mathrm{d} u_{t}(\theta)}{\mathrm{d} t} .
$$

For $\phi \in C^{*}=C\left([0,1],\left(R^{2}\right)^{*}\right)$, the adjoint operator $A^{*}$ of $A$ is defined by

$$
A^{*}(0) \phi(s)= \begin{cases}-\frac{\mathrm{d} \phi(s)}{\mathrm{d} s}, & s \in(0,1], \\ \int_{-1}^{0} \mathrm{~d} \eta^{T}(t, 0) \phi(-t), & s=0,\end{cases}
$$

where $\eta^{T}$ is the transpose of the matrix $\eta$. We define

$$
\begin{aligned}
\langle\phi, \varphi\rangle= & \bar{\phi}(0) \varphi(0) \\
& -\int_{-1}^{0} \int_{\xi=0}^{\theta} \bar{\phi}(\xi-\theta) \mathrm{d} \eta(\theta) \varphi(\xi) \mathrm{d} \xi
\end{aligned}
$$

where $\eta(\theta)=\eta(\theta, 0)$. We know that $i \omega_{0} \tau_{0}$ is an eigenvalue of $A(0)$, so $-i \omega_{0} \tau_{0}$ is also an eigenvalue of $A^{*}(0)$. We can get $q(\theta)=\left(1, q_{1}\right)^{T} e^{i \omega_{0} \tau_{0} \theta}$.

From the above discussion, it is easy to know that

$$
\begin{gathered}
A q(\theta)=i \omega_{0} \tau_{0} q(\theta), \\
\tau_{0} A_{1} q(0)+\tau_{0} B_{1} q(-1)=i \omega_{0} \tau_{0} q(0) .
\end{gathered}
$$

Hence, we obtain

$$
q_{1}=\frac{\alpha}{\left(i \omega_{0}+\gamma+\delta\right) e^{i \omega_{0} \tau_{0}}}
$$

Suppose that the eigenvector

$$
q^{*}(s)=\frac{1}{\rho}\left(1, q_{1}^{*}\right)^{T} e^{i \omega_{0} \tau_{0} s}
$$

Then the following relationship is obtained:

$$
A^{*} q^{*}(0)=-i \omega_{0} \tau_{0} q^{*}(0)
$$

Hence, we obtain

$$
q_{1}^{*}=\frac{1}{\left(\gamma+\delta-i \omega_{0}\right)}\left(\frac{2(\alpha+\delta)(\gamma+\delta)}{\alpha+\gamma+\delta}-\beta\right) .
$$

Let

$$
\begin{gathered}
\left\langle q^{*}, q\right\rangle=1, \\
\left\langle q^{*}, q\right\rangle=\bar{q}^{*}(0) q(0)-\int_{\theta=-1}^{0} \int_{\xi=0}^{\theta} \bar{q}^{* T}(\xi-\theta) \mathrm{d} \eta(\theta) \varphi(\xi) \mathrm{d} \xi \\
=\frac{1}{\rho}\left(1+q_{1} \bar{q}_{1}^{*}\right) \\
-\int_{\theta=-1}^{0} \int_{\xi=0}^{\theta} \tau_{0} \frac{1}{\rho}\left[1, \bar{q}_{1}^{*}\right]\left[A_{2} \delta(\theta)+B_{2} \delta(\theta+1)\right] \\
\quad \times\left[\begin{array}{c}
1 \\
q_{1}
\end{array}\right] e^{i \tau_{0} \omega_{0} \theta} \mathrm{d} \xi \mathrm{d} \theta \\
=\frac{1}{\rho}\left(1+q_{1} \bar{q}_{1}^{*}\right)+\tau_{0} \frac{1}{\rho} \alpha\left(1-\bar{q}_{1}^{*}\right) e^{-i \tau_{0} \omega_{0}}=1 .
\end{gathered}
$$

Hence, we obtain

$$
\rho=\left(1+q_{1} \bar{q}_{1}^{*}\right)+\tau_{0} \alpha\left(1-\bar{q}_{1}^{*}\right) e^{-i \tau_{0} \omega_{0}} .
$$

In the remainder of this section, by using the same notations as in the work by Hassard et al. [17], we first compute the coordinates $f$ or describing the center manifold $C_{0}$ at $\mu=0$. Letting $u_{t}$ be the solution of (18) with $\mu=0$, we define $z(t)=\left\langle q^{*}, u_{t}\right\rangle$, and

$$
W(t, \theta)=u_{t}-2 \operatorname{Re}\{z(t) q(\theta)\} .
$$

On the center manifold $C_{0}$ we have

$$
W(t, \theta)=W(z, \bar{z}, t)
$$

where

$$
W(z, \bar{z}, t)=W_{20}(\theta) \frac{z^{2}}{2}+W_{11}(\theta) \frac{z \bar{z}}{2}+W_{02}(\theta) \frac{\bar{z}^{2}}{2}+\cdots
$$

In fact, $z$ and $\bar{z}$ are local coordinates for $C_{0}$ in the direction of $q$ and $q^{*}$. Note that if $u_{t}$ is, we will deal with real solutions only. Since $\mu=0$,

$$
\begin{aligned}
\dot{z}(t) & =\left\langle q^{*}, u_{t}\right\rangle=\left\langle q^{*}, A(\mu) u_{t}+R(\mu) u_{t}\right\rangle \\
& =\left\langle q^{*}, A u_{t}\right\rangle+\left\langle q^{*}, R u_{t}\right\rangle \\
& =i \tau_{0} \omega_{0} z+\bar{q}^{*}(0) \cdot f(0, W(t, 0)+2 \operatorname{Re}[z(t) q(0)]) .
\end{aligned}
$$

Rewrite (37) as

$$
\dot{z}(t)=i \tau_{0} \omega_{0} z+g(z, \bar{z})
$$

where

$$
g(z, \bar{z})=g_{20} \frac{z^{2}}{2}+g_{11} z \bar{z}+g_{02} \frac{\bar{z}^{2}}{2}+g_{21} \frac{z^{2} \bar{z}}{2}+\cdots
$$




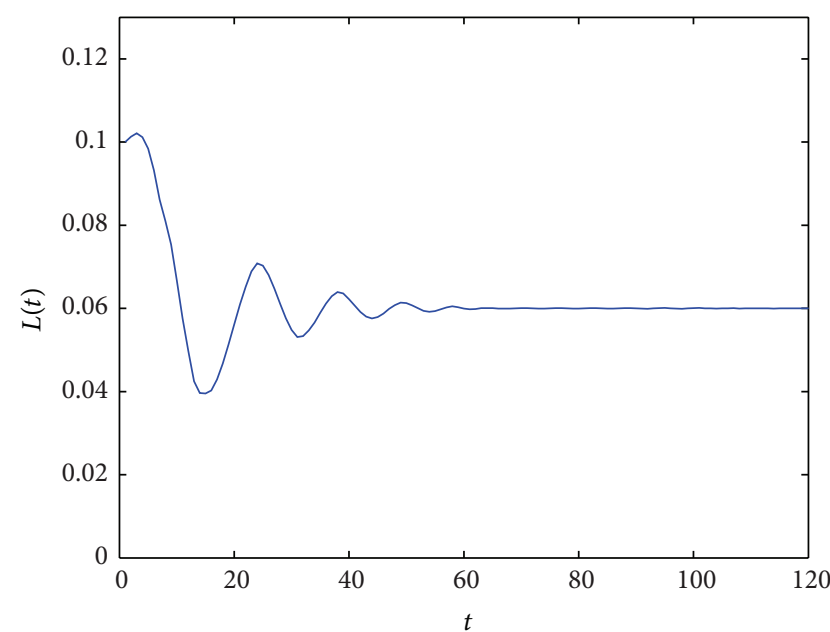

(a)

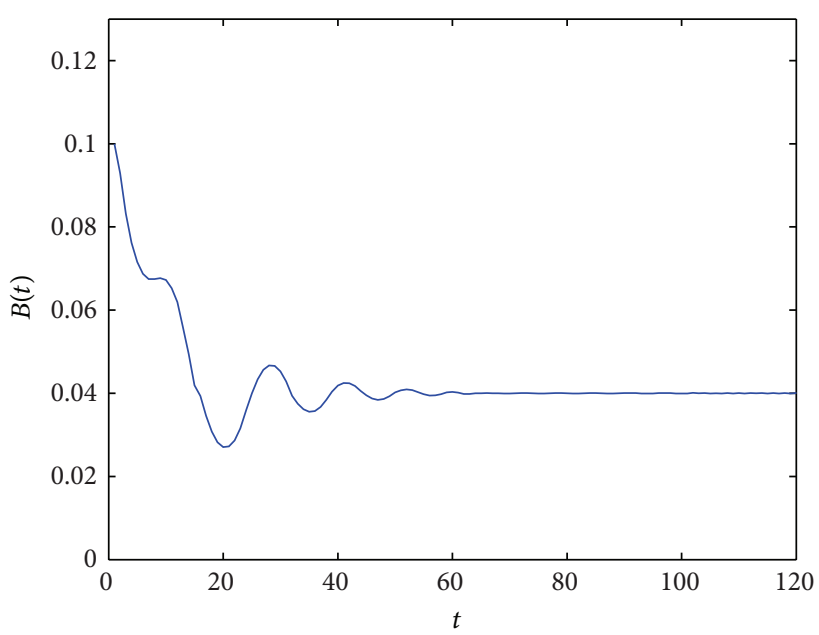

(b)

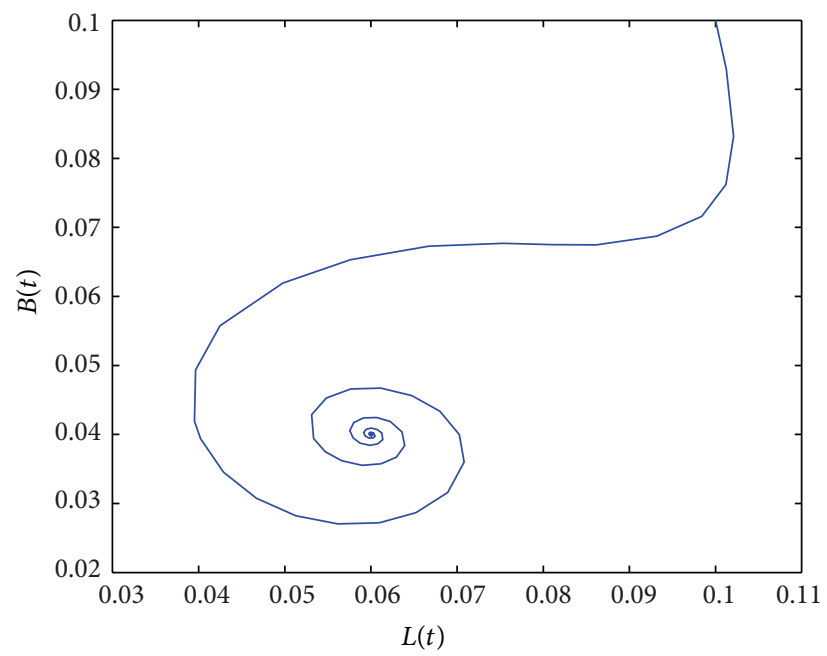

(c)

FIgURE 1: The positive equilibrium $E_{*}$ of system (3) is asymptotically stable.

From (18) and (38), we have

$$
\begin{aligned}
\dot{W} & =\dot{u}_{t}-\dot{z} q-\dot{\bar{z}} \bar{q} \\
& = \begin{cases}A W-2 \operatorname{Re}\left[\bar{q}^{*}(0) f(z, \bar{z}) q(\theta)\right], & -1 \leq \theta<0, \\
A W-2 \operatorname{Re}\left[\bar{q}^{*}(0) f(z, \bar{z}) q(\theta)\right]+f, & \theta=0\end{cases}
\end{aligned}
$$

Let

$$
\dot{W}=A W+H(z, \bar{z}, \theta),
$$

where

$$
H(z, \bar{z}, \theta)=H_{20}(\theta) \frac{z^{2}}{2}+H_{11}(\theta) z \bar{z}+H_{02}(\theta) \frac{\bar{z}^{2}}{2}+\cdots
$$

Taking the derivative of $W$ with respect to $t$ in (36), we have

$$
\dot{W}=W_{z} \dot{z}+W_{\bar{z}} \dot{\bar{z}}
$$

Substituting (36) and (38) into (43), we obtain

$$
\begin{aligned}
\dot{W}= & \left(W_{20} z+W_{11} \bar{z}+\cdots\right)\left(i \tau_{0} \omega_{0} z+g\right) \\
& +\left(W_{11} z+W_{02} \bar{z}+\cdots\right)\left(-i \tau_{0} \omega_{0} \bar{z}+\bar{g}\right) .
\end{aligned}
$$

Then substituting (36) and (41) into (42), we have the following results:

$$
\begin{aligned}
\dot{W}= & \left(A W_{20}+H_{20}\right) \frac{z^{2}}{2}+\left(A W_{11}+H_{11}\right) z \bar{z} \\
& +\left(A W_{02}+H_{02}\right) \frac{\bar{z}^{2}}{2}+\cdots .
\end{aligned}
$$

Comparing the coefficients of (44) with (45), the following equations hold:

$$
\begin{gathered}
\left(A-2 i \tau_{0} \omega_{0}\right) W_{20}(\theta)=-H_{20}(\theta), \\
A W_{11}(\theta)=-H_{11}(\theta) .
\end{gathered}
$$




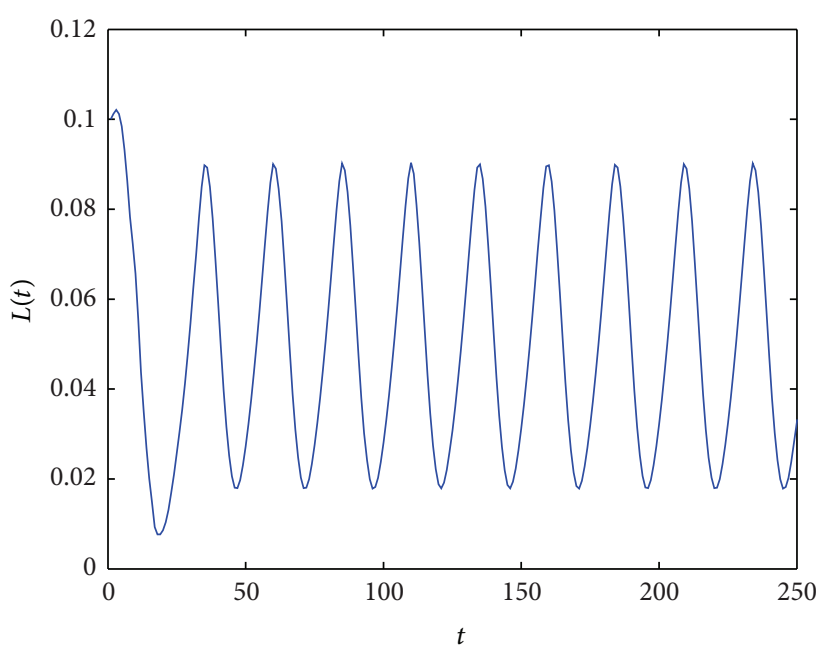

(a)

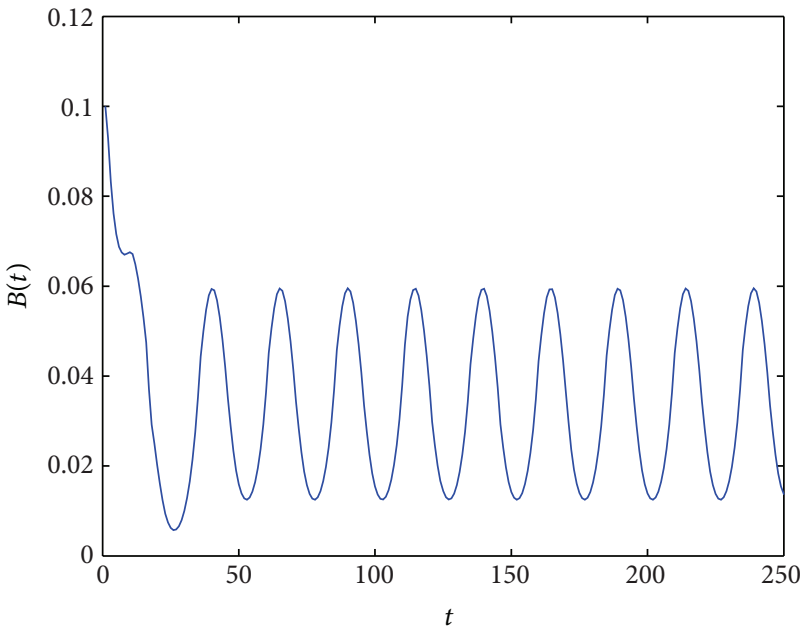

(b)

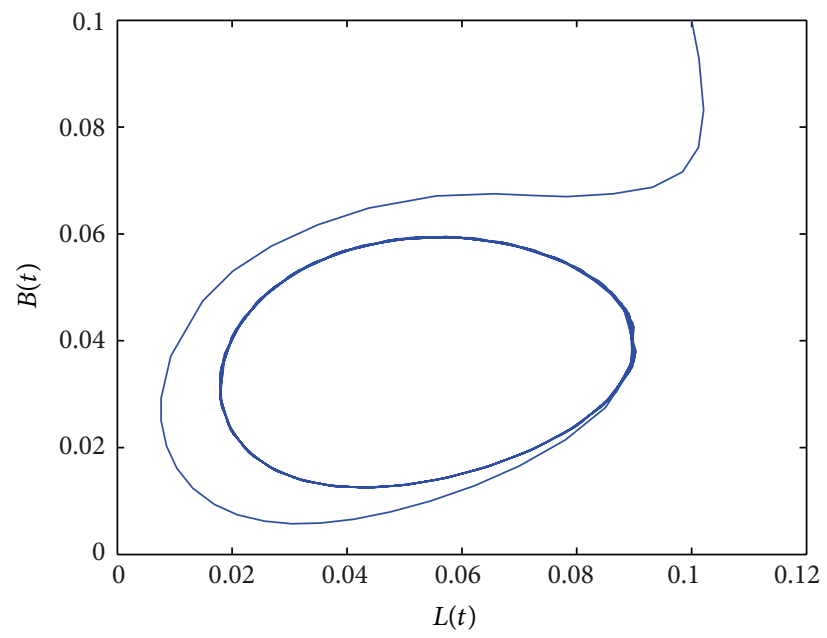

(c)

FIgURE 2: The bifurcation periodic solution is stable.

Since $u_{t}=u(t+\theta)=W(z, \bar{z}, \theta)+z q+\bar{z} \cdot \bar{q}$, we have

$$
u_{t}=\left(\begin{array}{l}
W^{(1)}(z, \bar{z}, \theta) \\
W^{(2)}(z, \bar{z}, \theta)
\end{array}\right)+z\left(\begin{array}{c}
1 \\
q_{1}
\end{array}\right) e^{i \omega_{0} \theta}+\bar{z}\left(\begin{array}{c}
1 \\
\bar{q}_{1}
\end{array}\right) e^{-i \omega_{0} \theta}
$$

Thus, we can obtain

$$
\begin{gathered}
\varphi_{1}(0)=z+\bar{z}+W_{20}^{(1)}(0) \frac{z^{2}}{2}+W_{11}^{(1)}(0) z \bar{z}+W_{02}^{(1)}(0) \frac{\bar{z}^{2}}{2}, \\
\varphi_{2}(0)=z q_{1}+\bar{z} \bar{q}_{1}+W_{20}^{(2)}(0) \frac{z^{2}}{2}+W_{11}^{(2)}(0) z \bar{z}+W_{02}^{(2)}(0) \frac{\bar{z}^{2}}{2} .
\end{gathered}
$$

So, we have

$$
\begin{aligned}
\varphi_{1}(0) \varphi_{2}(0)= & q_{1} z^{2}+\bar{q}_{1} \bar{z}^{2}+\left(q_{1}+\bar{q}_{1}\right) z \bar{z} \\
& +\left(W_{11}^{(2)}+\frac{1}{2} W_{20}^{(2)}+W_{11}^{(1)} q_{1}+\frac{1}{2} W_{20}^{(1)} \bar{q}_{1}\right) z^{2} \bar{z}
\end{aligned}
$$

$$
\begin{gathered}
\varphi_{1}^{2}(0)=z^{2}+\bar{z}^{2}+2 z \bar{z}+\left(W_{20}^{(1)}+2 W_{11}^{(1)}\right) z^{2} \bar{z} \\
\varphi_{2}^{2}(0)=q_{1}^{2} z^{2}+\bar{q}_{1}^{2} \bar{z}^{2}+2 q_{1} \bar{q}_{1} z \bar{z}+\left(\bar{q}_{1} W_{20}^{(2)}+2 q_{1} W_{11}^{(2)}\right) z^{2} \bar{z} .
\end{gathered}
$$

It follows from (38) and (39) that

$$
f(z, \bar{z})=\left(\begin{array}{c}
K_{1} z^{2}+K_{2} z \bar{z}+K_{3} \bar{z}^{2}+K_{4} z^{2} \bar{z} \\
0
\end{array}\right),
$$

where

$$
\begin{gathered}
K_{1}=-\beta \tau_{0}\left(1+q_{1}^{2}+2 q_{1}\right), \\
K_{2}=-\beta \tau_{0}\left(2+2 q_{1} \bar{q}_{1}+2 q_{1}+2 \bar{q}_{1}\right), \\
K_{3}=-\beta \tau_{0}\left(1+\bar{q}_{1}^{2}+2 \bar{q}_{1}\right), \\
K_{4}=-\beta \tau_{0}\left(2 W_{11}^{(2)}+W_{20}^{(2)}+2 W_{11}^{(1)} q_{1}+W_{20}^{(1)} \bar{q}_{1}\right. \\
\left.+W_{20}^{(1)}+2 W_{11}^{(1)}+\bar{q}_{1} W_{20}^{(2)}+2 q_{1} W_{11}^{(2)}\right) .
\end{gathered}
$$




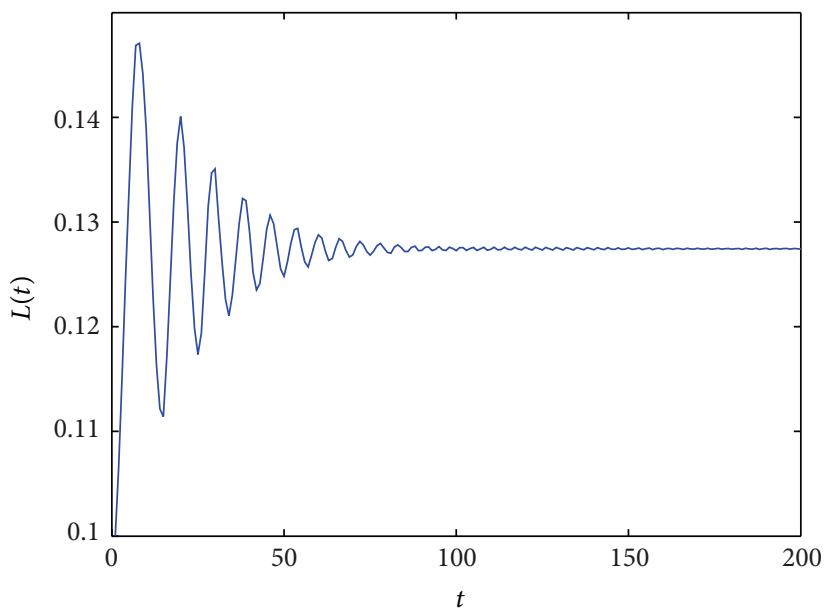

(a)

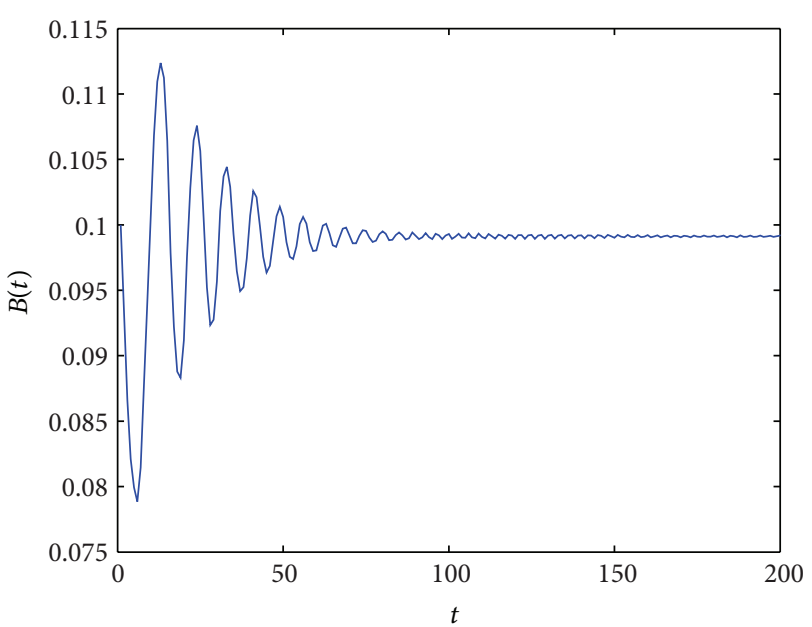

(b)

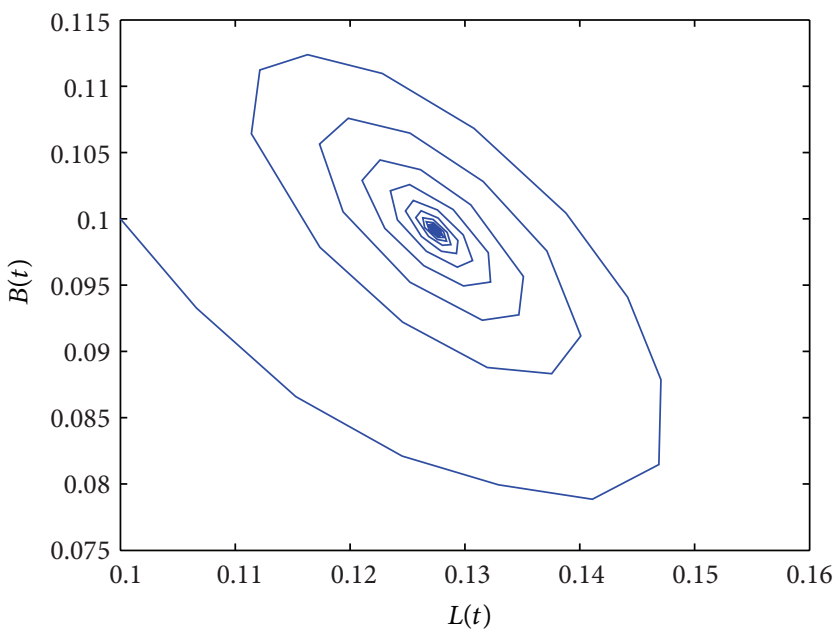

(c)

Figure 3: The positive equilibrium $E_{*}$ of system is asymptotically stable.

Then we have

$$
g(z, \bar{z})=\frac{1}{\rho}\left(1, \bar{q}_{1}^{*}\right)\left(\begin{array}{c}
K_{1} z^{2}+K_{2} z \bar{z}+K_{3} \bar{z}^{2}+K_{4} z^{2} \bar{z} \\
0
\end{array}\right) .
$$

Comparing the coefficients of the above equation with those in (41), we have

$$
\begin{array}{ll}
g_{20}=\frac{1}{\rho} K_{1}, & g_{11}=\frac{1}{\rho} K_{2}, \\
g_{02}=\frac{1}{\rho} K_{3}, & g_{21}=\frac{1}{\rho} K_{4} .
\end{array}
$$

In what follows, we focus on the computation of $W_{20}(\theta)$ and $W_{11}(\theta)$. For the expression of $g_{21}$, we have

$$
\begin{aligned}
H(z, \bar{z}, \theta)= & -2 \operatorname{Re}\left(\bar{q}^{*}(0) f(z, \bar{z}) q(\theta)\right)+R u_{t} \\
= & -\left(\frac{1}{2} g_{20} z^{2}+g_{11} z \bar{z}+\frac{1}{2} g_{02} \bar{z}^{2}+\cdots\right) q(\theta) \\
& -\left(\frac{1}{2} \bar{g}_{20} \bar{z}^{2}+\bar{g}_{11} z \bar{z}+\frac{1}{2} \bar{g}_{02} z^{2}+\cdots\right) \bar{q}(\theta) \\
& +R u_{t} .
\end{aligned}
$$

Comparing the coefficients of the above equation, we can obtain that

$$
\begin{array}{ll}
H_{20}(\theta)=-g_{20} q(\theta)-\bar{g}_{02} \bar{q}(\theta), & \theta \in[-1,0), \\
H_{11}(\theta)=-g_{11} q(\theta)-\bar{g}_{11} \bar{q}(\theta), & \theta \in[-1,0) .
\end{array}
$$

Substituting (56) into (46) and (57) into (47), respectively, we get

$$
\begin{gathered}
\dot{W}_{20}(\theta)=2 i \tau_{0} \omega_{0} W_{20}(\theta)+g_{20} q(\theta)+\bar{g}_{02} \bar{q}(\theta), \\
\dot{W}_{11}(\theta)=g_{11} q(\theta)+\bar{g}_{11} \bar{q}(\theta) .
\end{gathered}
$$

We can easily obtain the solutions of (58) as

$$
\begin{aligned}
W_{20}(\theta)= & \frac{i g_{20}}{\tau_{0} \omega_{0}} q(0) e^{i \tau_{0} \omega_{0} \theta}-\frac{\bar{g}_{02}}{3 i \tau_{0} \omega_{0}} \bar{q}(0) e^{-i \tau_{0} \omega_{0} \theta} \\
& +E_{1} e^{2 i \tau_{0} \omega_{0} \theta}
\end{aligned}
$$

$\dot{W}_{11}(\theta)=\frac{g_{11}}{i \tau_{0} \omega_{0}} q(0) e^{i \tau_{0} \omega_{0} \theta}-\frac{\bar{g}_{11}}{i \tau_{0} \omega_{0}} \bar{q}(0) e^{-i \tau_{0} \omega_{0} \theta}+E_{2}$. 


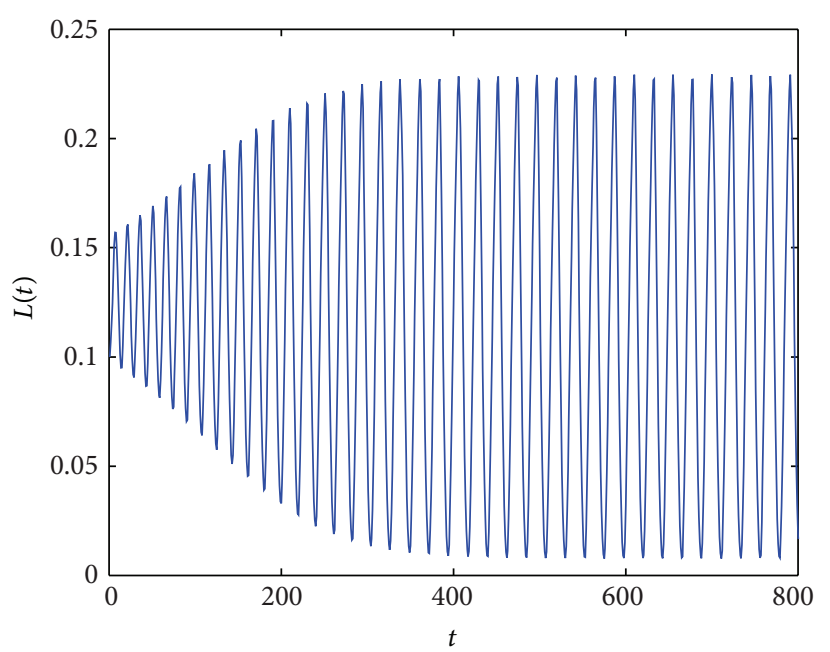

(a)

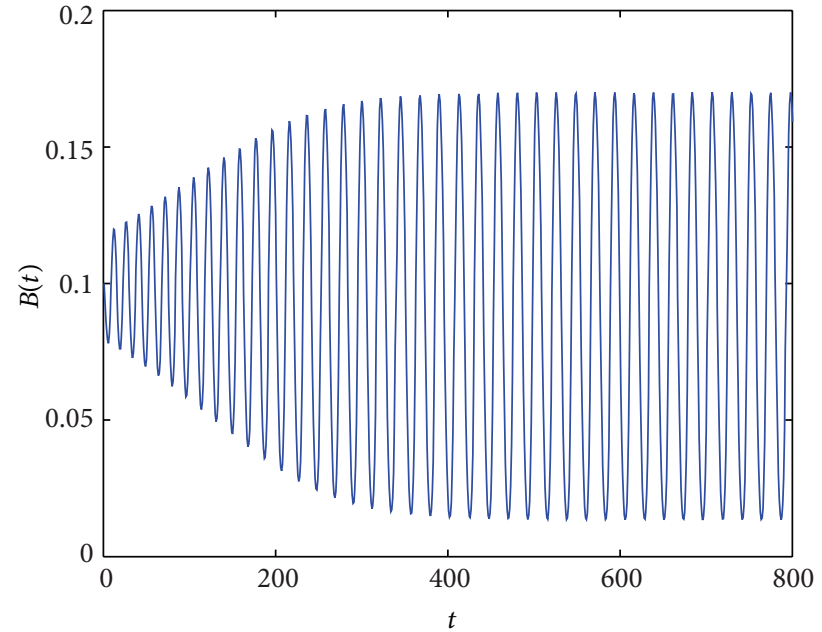

(b)

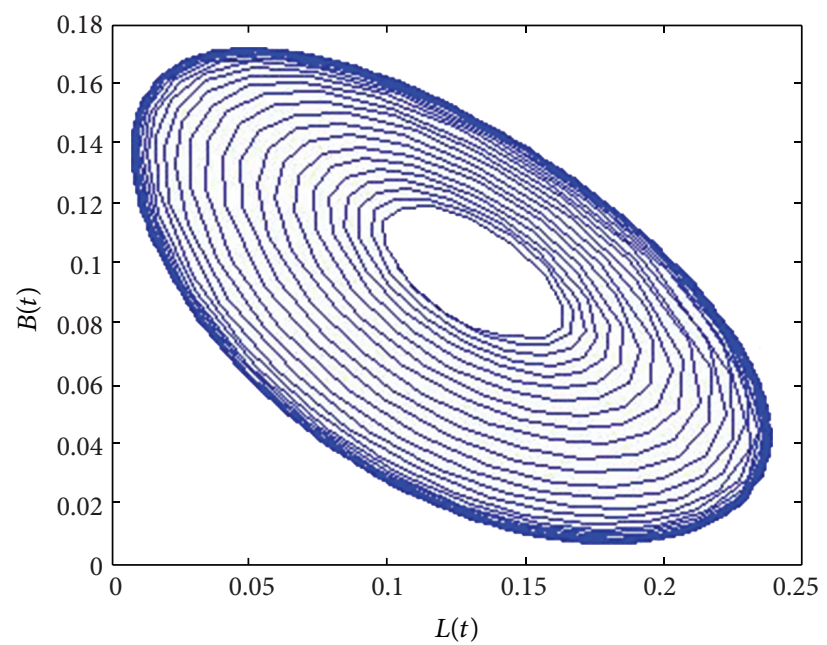

(c)

Figure 4: The bifurcation periodic solution is stable.

We will determine $E_{1}$ and $E_{2}$. Form the definition of $A$ in (23), we have

$$
\begin{gathered}
\int_{-1}^{0} d \eta(\theta) W_{20}(0)=2 i \tau_{0} \omega_{0} W_{20}(0)-H_{20}(0), \\
\int_{-1}^{0} d \eta(\theta) W_{11}(0)=-H_{11}(0) .
\end{gathered}
$$

From (59), (56) and (57), we have

$$
\begin{aligned}
& H_{20}(\theta)=-g_{20} q(\theta)-\bar{g}_{02} \bar{q}(\theta)+\left(K_{1}, 0\right)^{T}, \\
& H_{11}(\theta)=-g_{11} q(\theta)-\bar{g}_{11} \bar{q}(\theta)+\left(K_{2}, 0\right)^{T} .
\end{aligned}
$$

Substituting (59) and (61) into (62) and noticing that

$$
\begin{gathered}
\left(i \omega_{0} I-\int_{-1}^{0} e^{i \omega_{0} \theta} d \eta(\theta)\right) q(0)=0, \\
\left(-i \omega_{0} I-\int_{-1}^{0} e^{-i \omega_{0} \theta} d \eta(\theta)\right) \bar{q}(0)=0,
\end{gathered}
$$

we can obtain

$$
\left(2 i \omega_{0} I-\int_{-1}^{0} e^{2 i \tau_{0} \omega_{0} \theta} d \eta(\theta)\right) E_{1}=\left(K_{1}, 0\right)^{T},
$$

which leads to

$$
\begin{aligned}
& \left(\begin{array}{cc}
2 i \omega_{0}-\beta-\frac{2(\alpha+\delta)(\gamma+\delta)}{\alpha+\gamma+\delta}+\delta+\alpha e^{-2 i \omega_{0} \tau_{0}} & \beta-\frac{2(\alpha+\delta)(\gamma+\delta)}{\alpha+\gamma+\delta} \\
-\alpha e^{-2 i \omega_{0} \tau_{0}} & 2 i \omega_{0}+\gamma+\delta
\end{array}\right) E_{1} \\
& =\left(\begin{array}{c}
K_{1} \\
0
\end{array}\right), \\
& \left(\begin{array}{cc}
-\beta-\frac{2(\alpha+\delta)(\gamma+\delta)}{\alpha+\gamma+\delta}+\delta+\alpha & \beta-\frac{2(\alpha+\delta)(\gamma+\delta)}{\alpha+\gamma+\delta} \\
-\alpha & \gamma+\delta
\end{array}\right) E_{2}=\left(\begin{array}{c}
K_{2} \\
0
\end{array}\right) .
\end{aligned}
$$


It follows that

$$
\begin{aligned}
E_{1}= & \left(\begin{array}{cc}
2 i \omega_{0}-\beta-\frac{2(\alpha+\delta)(\gamma+\delta)}{\alpha+\gamma+\delta}+\delta+\alpha e^{-2 i \omega_{0} \tau_{0}} & \beta-\frac{2(\alpha+\delta)(\gamma+\delta)}{\alpha+\gamma+\delta} \\
-\alpha e^{-2 i \omega_{0} \tau_{0}} & 2 i \omega_{0}+\gamma+\delta
\end{array}\right)^{-1} \\
& \times\left(\begin{array}{c}
K_{1} \\
0
\end{array}\right), \\
E_{2}= & \left(\begin{array}{cc}
-\beta-\frac{2(\alpha+\delta)(\gamma+\delta)}{\alpha+\gamma+\delta}+\delta+\alpha & \beta-\frac{2(\alpha+\delta)(\gamma+\delta)}{\alpha+\gamma+\delta} \\
-\alpha & \gamma+\delta
\end{array}\right)^{-1}\left(\begin{array}{c}
K_{2} \\
0
\end{array}\right) .
\end{aligned}
$$

Hence, we know $W_{20}$ and then we can obtain $g_{21}$. The following parameters can be calculated:

$$
\begin{gathered}
C_{1}(0)=\frac{i}{2 \omega_{0} \tau_{0}}\left(g_{20} g_{11}-2\left|g_{11}\right|^{2}-\frac{1}{3}\left|g_{02}\right|^{2}\right)+\frac{g_{21}}{2}, \\
\mu_{2}=-\frac{\operatorname{Re}\left\{C_{1}(0)\right\}}{\operatorname{Re}\left\{\lambda^{\prime}\left(\tau_{0}\right)\right\}}, \quad \beta_{2}=2 \operatorname{Re}\left\{C_{1}(0)\right\}, \\
T_{2}=-\frac{\operatorname{Im}\left\{C_{1}(0)\right\}+\mu_{2} \operatorname{Im}\left\{\lambda^{\prime}\left(\tau_{0}\right)\right\}}{\tau_{0} \omega_{0}} .
\end{gathered}
$$

Theorem 3. Under the condition of Theorem 1, one has the following.

(1) $\mu=0$ is Hopf bifurcation value of system (18).

(2) The direction of Hopf bifurcation is determined by the sign of $\mu_{2}$ : if $\mu_{2}>0$, the Hopf bifurcation is supercritical; if $\mu_{2}<0$, the Hopf bifurcation is subcritical.

(3) The stability of bifurcating periodic solutions is determined by $\beta_{2}$ : if $\beta_{2}<0$, the periodic solutions are stable; if $\beta_{2}>0$, they are unstable.

\section{Numerical Examples}

In this section, some numerical examples of system (3) are presented to justify the previous theorem above.

Example 1. Consider system (3) with parameters $\alpha=0.8, \beta=$ $0.8, \gamma=0.8$, and $\delta=0.4$. Then $R_{0}=1.1111, E_{*}=$ $(0.06,0.04)$, and (7) has one positive real root $\omega=0.3470$. It follows by (11) that $\tau_{0}=3.5705$. First, we choose $\tau=3<\tau_{0}$. For a set of initial conditions satisfying $L(0)=0.1$ and $B(0)=$ 0.1 , Figure 1 demonstrates the evolutions from which it can be seen that the equilibrium is asymptotically stable. Second, we choose $\tau=3.7>\tau_{0}$. For a set of initial conditions satisfying, the corresponding wave form and phase plots are shown in Figure 2, from which it is easy to see that a Hopf bifurcation occurs.

Example 2. Consider system (3) with parameters $\alpha=$ $0.7, \beta=0.8, \gamma=0.5$, and $\delta=0.4$. Then $R_{0}=1.2929, E_{*}=$ $(0.1274,0.0991)$, and (7) has one positive real root $\omega=$ 0.4463 . It follows by (11) that $\tau_{0}=4.0204$. First, we choose $\tau=3.3<\tau_{0}$. For a set of initial conditions satisfying
$L(0)=0.1$ and $B(0)=0.1$, Figure 3 demonstrates the evolutions from which it can be seen that the equilibrium is asymptotically stable. Second, we choose $\tau=4.2>\tau_{0}$. For a set of initial conditions satisfying $L(0)=0.1$ and $B(0)=0.1$, the corresponding wave form and phase plots are shown in Figure 4, from which it is easy to see that a Hopf bifurcation occurs.

\section{Conclusions}

In this paper, we have constructed a computer virus model with time delay depending on the SLBS model. The theoretical analyses for the computer virus models are given. Furthermore, it is proved that there exists a Hopf bifurcation when time crosses through the critical value. Finally, the numerical simulations illustrate our results.

\section{Acknowledgments}

The authors were greatly indebted to the anonymous reviewers for their valuable suggestions. This paper was supported by the National Natural Science Foundation of China (no. 61170320), the Natural Science Foundation of Guangdong Province (no. S2011040002981), and Nature Science Foundation of Guangdong Medical College (B2012053).

\section{References}

[1] J. O. Kephart, S. R. White, and D. M. Chess, "Computers and epidemiology," IEEE Spectrum, pp. 20-26, 1993.

[2] F. Cohen, "Computer viruses. Theory and experiments," Computers and Security, vol. 6, no. 1, pp. 22-35, 1987.

[3] X. Han and Q. Tan, "Dynamical behavior of computer virus on Internet," Applied Mathematics and Computation, vol. 217, no. 6, pp. 2520-2526, 2010.

[4] B. K. Mishra and N. Jha, "Fixed period of temporary immunity after run of anti-malicious software on computer nodes," Applied Mathematics and Computation, vol. 190, no. 2, pp. 12071212, 2007.

[5] B. K. Mishra and S. K. Pandey, "Fuzzy epidemic model for the transmission of worms in computer network," Nonlinear Analysis: Real World Applications, vol. 11, no. 5, pp. 4335-4341, 2010.

[6] J. Ren, X. Yang, L.-X. Yang, Y. Xu, and F. Yang, "A delayed computer virus propagation model and its dynamics," Chaos, Solitons and Fractals, vol. 45, no. 1, pp. 74-79, 2012.

[7] J. Ren, X. Yang, Q. Zhu, L.-X. Yang, and C. Zhang, "A novel computer virus model and its dynamics," Nonlinear Analysis: Real World Applications, vol. 13, no. 1, pp. 376-384, 2012.

[8] B. K. Mishra and S. K. Pandey, "Dynamic model of worms with vertical transmission in computer network," Applied Mathematics and Computation, vol. 217, no. 21, pp. 8438-8446, 2011.

[9] H. Yuan and G. Chen, "Network virus-epidemic model with the point-to-group information propagation," Applied Mathematics and Computation, vol. 206, no. 1, pp. 357-367, 2008.

[10] B. K. Mishra and D. K. Saini, "SEIRS epidemic model with delay for transmission of malicious objects in computer network," Applied Mathematics and Computation, vol. 188, no. 2, pp. 14761482, 2007. 
[11] F. Wang, Y. Zhang, C. Wang, J. Ma, and S. Moon, "Stability analysis of a SEIQV epidemic model for rapid spreading worms," Computers and Security, vol. 29, no. 4, pp. 410-418, 2010.

[12] B. K. Mishra and N. Jha, "SEIQRS model for the transmission of malicious objects in computer network," Applied Mathematical Modelling, vol. 34, no. 3, pp. 710-715, 2010.

[13] X. Yang and L.-X. Yang, "Towards the epidemiological modeling of computer viruses," Discrete Dynamics in Nature and Society, vol. 2012, Article ID 259671, 11 pages, 2012.

[14] L.-X. Yang, X. Yang, L. Wen, and J. Liu, "A novel computer virus propagation model and its dynamics," International Journal of Computer Mathematics, 2012.

[15] L.-X. Yang, X. Yang, Q. Zhu, and L. Wen, "A computer virus model with graded cure rates," Nonlinear Analysis: Real World Applications, 2012.

[16] N. F. Britten, Essential Mathematical Biology, Springer, 2003.

[17] B. D. Hassard, N. D. Kazarinoff, and Y. H. Wan, Theory and Applications of Hopf Bifurcation, vol. 41, Cambridge University Press, Cambridge, UK, 1981.

[18] H. L. Freedman and V. Sree Hari Rao, "The trade-off between mutual interference and time lags in predator-prey systems," Bulletin of Mathematical Biology, vol. 45, no. 6, pp. 991-1004, 1983.

[19] J. K. Hale, Theory of Functional Differential Equations, Springer, New York, NY, USA, 1977. 


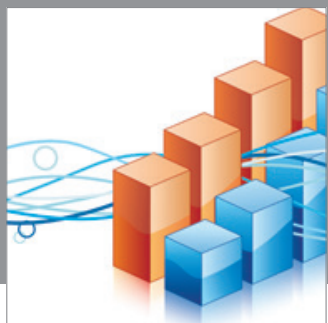

Advances in

Operations Research

mansans

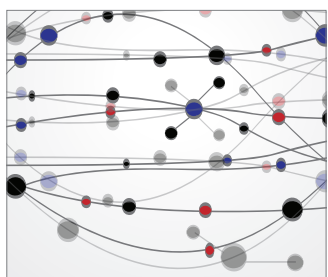

The Scientific World Journal
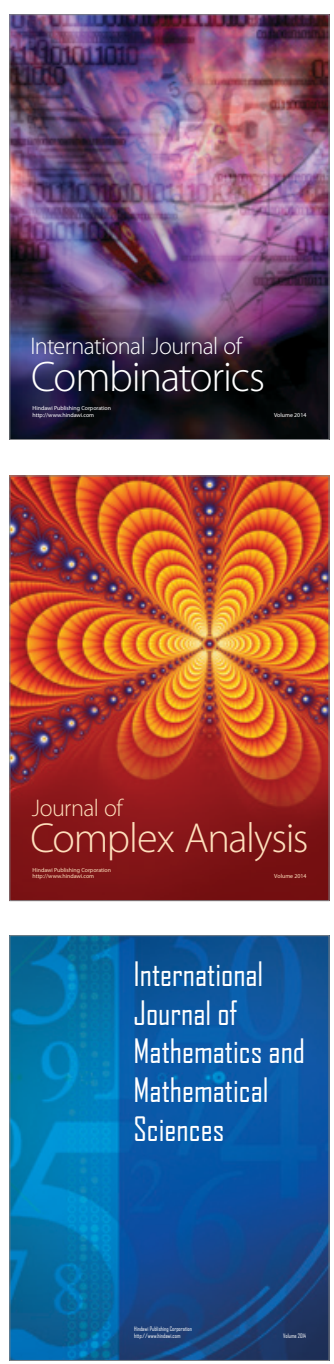
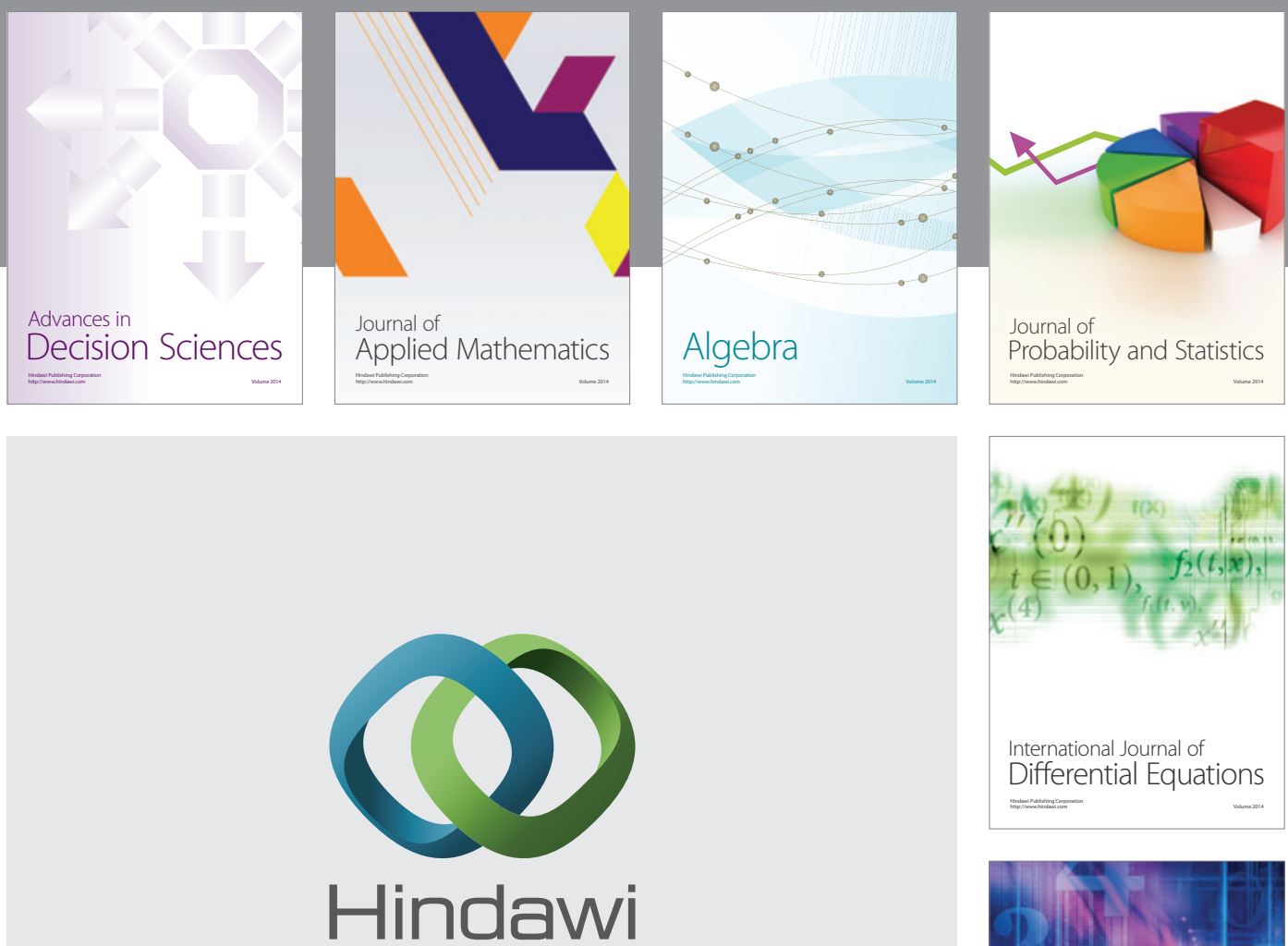

Submit your manuscripts at http://www.hindawi.com
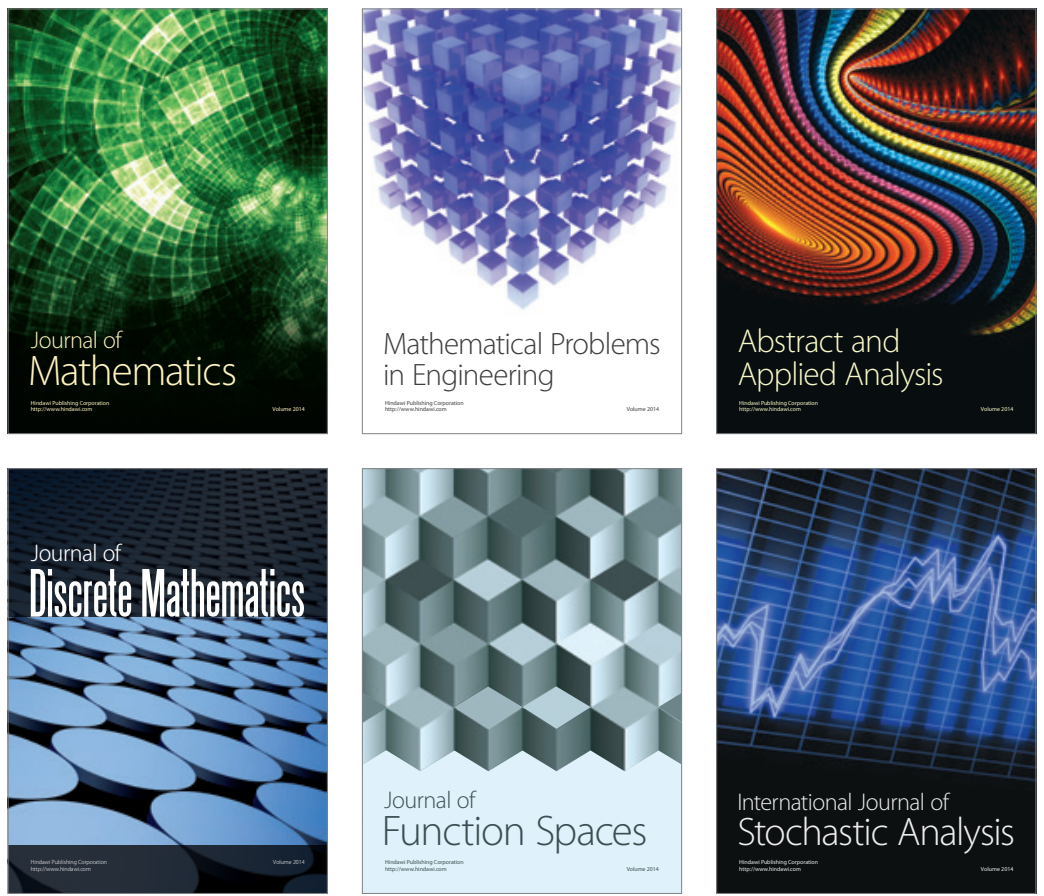

Journal of

Function Spaces

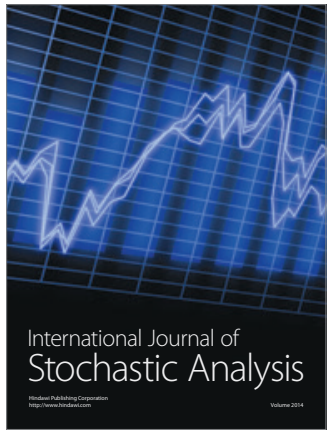

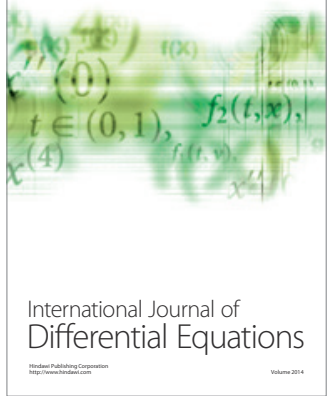
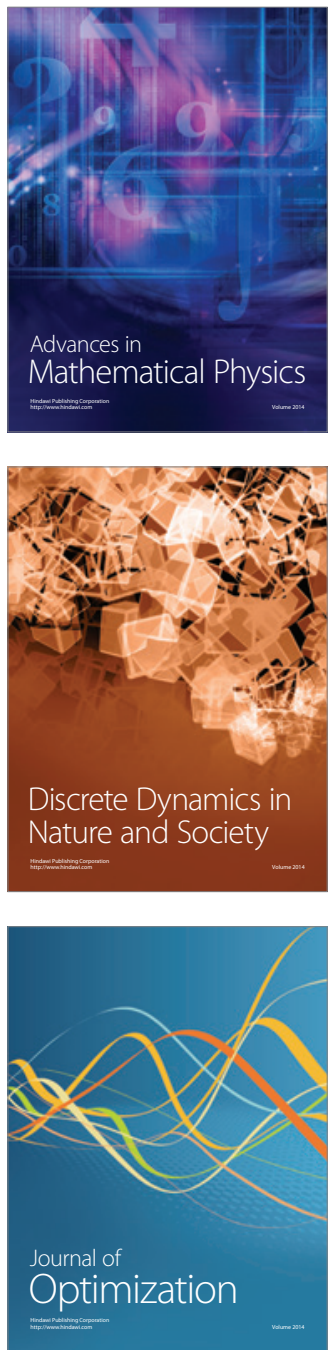